\title{
Linear bounded automata and rewrite systems : Influence of initial configurations on decision properties 1
}

\author{
A-C Caron \\ Université de Lille-Flandres-Artois \\ LIFL (URA 369-CNRS) UFR IEEA, Bâtiment M3 \\ 59655 Villeneuve d'Ascq Cedex France \\ mail: caronc@lifl.lifl.fr
}

\begin{abstract}
We prove that temination is undecidable for non-length-increasing string rewriting systems, using linear-bounded automata. On the other hand, we prove the undecidability of confluence for terminating rewriting systems when terms begin by a fixed symbol. These two results illustrate that sometimes restriction of problem to recognizable domains modify decidability properties, sometimes it does not (We only consider finite terms).
\end{abstract}

\section{Introduction}

With two problems, we prove the influence of initial configurations on rewriting decision properties. The first problem concerns termination, and the second, confluence.

Termination problems are fundamental in rewriting because they correspond to program termination for all data [Dershowitz \& Jouannaud]. Many termination criteria have been studied [Dershowitz] but, generally, termination is undecidable, even for one left-linear rule [Dauchet] or for a semi-Thue system [Huet \& Lankford]. Termination problems for one linear-rule or one rule on words remain open. But in this last case, if the rule is non-length-increasing, termination is clearly decidable.

Here, we prove undecidability of termination of non-length-increasing string rewriting systems (i.e. non-length-increasing semi-Thue systems). This problem is similar to linear-bounded automata termination [Book] and has been stated in the case of graphs by Litovsky and Metivier [Litovsky \& Metivier]. Therefore we revisit a paper of Hooper [Hooper], in which he studied termination of Turing machines and proved that termination is undecidable for linearbounded automata, and more generally, for Turing machines. Using technics suggested by Hooper, we prove directly undecidability of termination for non-length-increasing string rewriting systems.

In a first part, we construct a class of linear bounded automata whose termination is reduced to the Post correspondance problem. This result is well-known but we use our construction in the second part, and observe that undecidability subsists if we suppress the constraint of beginning from an initial configuration. In the third part, we want to bring a fact out: the link between decidability and recognizable restrictions on terms. Recognizable restriction means that terms belong to a recognizable language. Therefore, in opposition to this 
first result, we prove that confluence for terminating rewriting systems becomes undecidable if we restrict terms configuration to some recognizable set. (It is well-known that confluence is decidable for noetherian rewriting systems [Newman]). Confluence on recognizable tree languages is interesting because these languages are sorts (the finite automaton being the signature). Note that Otto proves that confluence on some congruence class is undecidable [Otto] but congruence classes are generally not recognizable.

\section{I - Termination of linear-bounded automata}

Linear-bounded automata have been created by Myhill [Myhill] and very studied since [Kuroda]. In particular, Hooper studied the undecidability of termination of Turing machine and linear-bounded automata [Hooper]. He called this problem immortality problem. Moreover, Hopcroft and Ullman showed that to every linear-bounded automaton, we can associate an equivalent terminating linear-bounded automaton [Hopcroft \& Ullman].

In this part, we prove directly the undecidability of termination for a class of linear-bounded automata which restore their initial configuration when they do not stop, using a suitable construction for the more general result of the second part.

Definition 1-1 : A machine terminates if and only if it stops for all data.

Definition 1-2 : A linear-bounded automaton (LBA) can be seen as a particular Turing machine. Its tape is an input/output tape whose length is linearly dependent of data length. A LBA is a sextuple $\left(\Sigma, \Gamma, \mathrm{Q}, \mathrm{Q}_{0}, \mathrm{Q}_{\mathrm{f}}, \Delta\right)$, where $\Sigma$ is the data alphabet, $\Gamma$ the work alphabet, $\mathrm{Q}$ is the states set, $\mathrm{Q}_{0}$ the initial states set, $\mathrm{Q}_{\mathrm{f}}$ the final states set, $\Delta$ is the next-move function. We suppose that the tape has the form \#<d $>\#$, where \#,<>> are never modified and $d$ is the data.

Vocabulary : We use Turing machines notions : instantaneous description, initial configuration, computation step, computation. More precisely :

- an instantaneous description (denoted ID) is a writing \# $<m_{1} q a m_{2}>\#$. It means that the head is reading the letter $a$, the word $m_{1}$ is on the left and $m_{2}$ is on the right, and $q$ is the machine state. - an initial configuration is an instantaneous description \#<qum $>\#$ where $q$ is an initial state.

- a step computation $\mathrm{ID}_{1} \rightarrow \mathrm{ID}_{2}$ means we can go from $\mathrm{ID}_{1}$ to $\mathrm{ID}_{2}$ with a transition of $\Delta$.

- a computation is a succession of computation steps from an initial configuration $\mathrm{ID}_{1}$ to a final configuration $\mathrm{ID}_{\mathrm{f}}$.

We are not interested in the result but in the computation stop. Therefore, final configuration is not important. But we need two notions : beginning of computation and sub-computation :

- a beginning of computation is a succession of computation steps, from an initial configuration $\mathrm{ID}_{1}$

$-\mathrm{ID}_{\mathrm{i}} \rightarrow \ldots \rightarrow \mathrm{ID}_{\mathrm{n}}$ is a sub-computation if there exists $\mathrm{ID}_{1} \rightarrow \ldots \rightarrow \mathrm{ID}_{\mathrm{i}} \rightarrow \ldots \rightarrow \mathrm{ID}_{\mathrm{n}}$ a beginning of computation which contains it.

Proposition 1-1 : [Hooper] Termination of linear-bounded automata is undecidable. 


\section{Post correspondance problem : [Post]}

The Post correspondance problem $P(\varphi, \psi)$ over an alphabet $X$ is given by two morphisms $\varphi$ and $\psi$ from $I^{*}$ to $X^{*} . P(\varphi, \psi)$ is solvable if and only if there exists $m_{I}$ in $I^{+}$such that $\varphi\left(m_{I}\right)=\psi\left(m_{I}\right)$. The Post correspondance problem is well-known undecidable.

We are working on a specific class of deterministic LBA, denoted $A_{\text {Post }}$, associated to the Post problem. A machine A $\varphi \psi$ of this class is associated to two morphisms $\varphi$ and $\psi$. If the tape of A $\varphi \Psi$ contains two words $m_{I}$ and $m_{X}$, such that $m_{X}=\varphi\left(m_{I}\right)=\psi\left(m_{I}\right)$ then the machine loops passing by its initial configuration again.

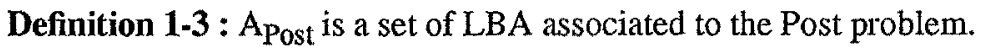

$A_{\text {Post }}=\left\{A \varphi \psi=\left(\Sigma, \Gamma, Q \varphi \psi,\left\{q_{0}\right\}, \varnothing, \Delta\right) \mid \varphi\right.$ and $\psi$ two morphisms from $I^{*}$ to $\left.X^{*}\right\}$, with

$. \Sigma=I \cup X \cup\{<,>\}, I$ and $X$ two disjoint finite alphabets.

$. \Gamma=\Sigma \cup \bar{I} \cup \bar{X}, \bar{X}$ and $\bar{I}$ constructed from $X$ and $I$ : for all $x$ in $X, \bar{X}$ is in $\bar{X}$ and for all $i$ in $I, \bar{i}$ is in $\bar{I}$.

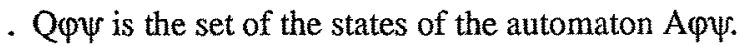

Appendix I contains in details the behaviour fonction $\Delta$ of $A \varphi \psi$. The following little program and the two examples explain the behaviour of a machine $A \varphi \Psi$

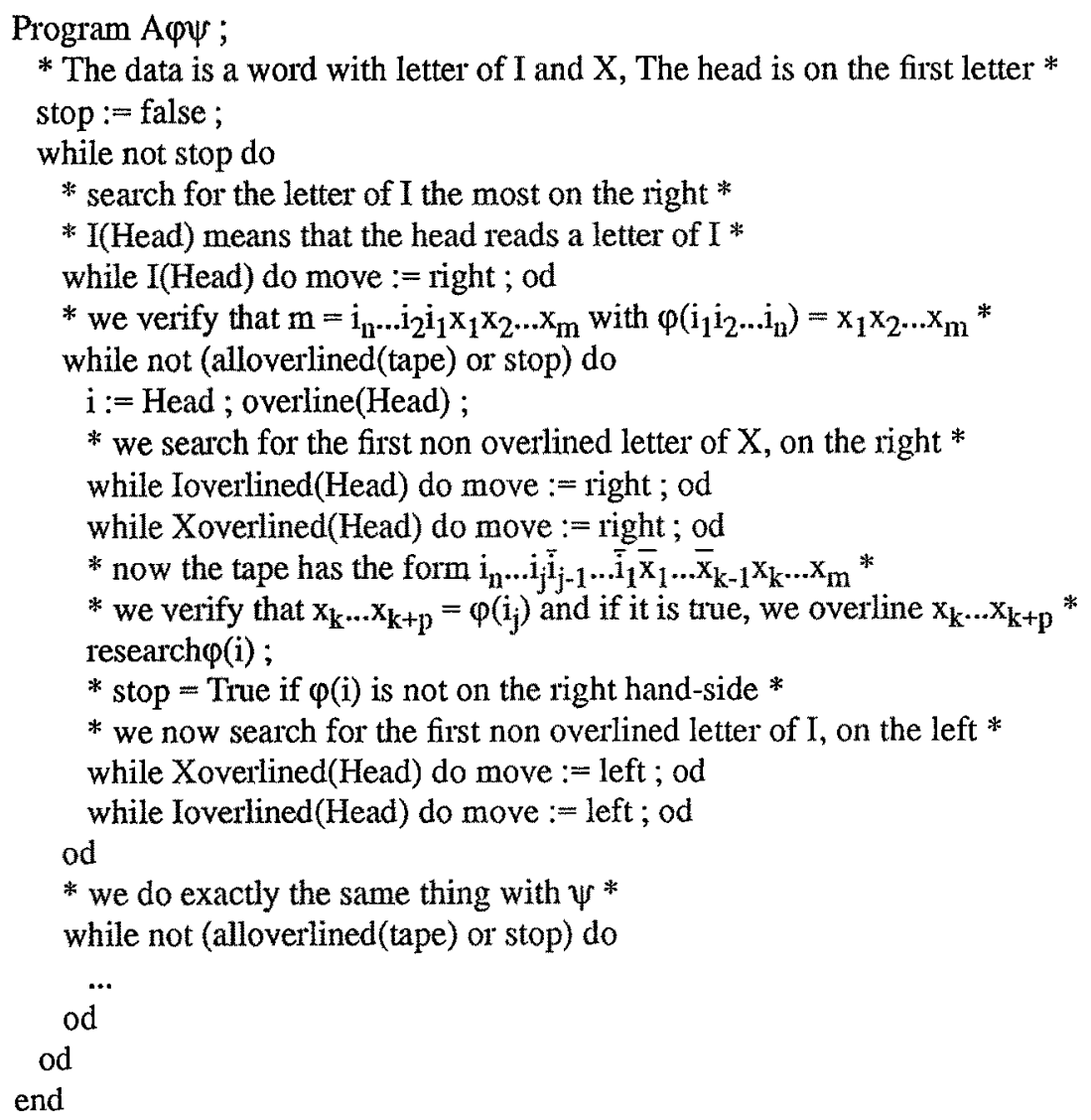




\section{Example 1:}

$\mathrm{I}=\{\mathrm{a}, \mathrm{b}\} ; \mathrm{X}=\{1,2,3\}$;

$\varphi(a)=123 ; \varphi(b)=32 ; \psi(a)=23 ; \psi(b)=321$.

- The tape contains the data ab32123. So the initial configuration is \#<qaab32123>\#

- The machine searches for the last non overlined letter of I. It is b.

The configuration is now \#<aq 2 b32123>\#.

- The machine overlines $\mathrm{b}$. The configuration is $\#<\mathrm{ab} \mathrm{q}_{\varphi(\mathrm{b}) 1} 32123>\#$

- The machine verifies that 3 is the first letter of $\varphi(b)$. Since it is true, it overlines the letter 1. The configuration is $\#<\mathrm{a} \overline{\mathrm{b} 3} \mathrm{q}_{\varphi(\mathrm{b}) 2} 2123>\#$.

- It verifies that 2 is the second letter of $\varphi(b)$ and overlines it. Since $\varphi(b)$ contains only two letters, the machine searches for the non overlined letter of I the most on the right. It is a.

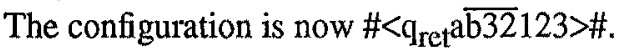

- The machine overlines a. The configuration is $\#<\bar{a} q_{\varphi(a) 1} \overline{b 32} 123>\#$.

-It searches for the first non overlined letter of X. It is 1 .

The configuration is \#<ab32 $q_{\varphi(a) 1} 123>\#$.

- It verifies that 1 is the first letter of $\varphi(a)$. Since it is true, it overlines it.

The configuration is \#<ab321 $q_{(\varphi(a) 2} 23>\#$.

- In the same way, the automaton overlines 2 and 3 , the second and the third letters of $\varphi(a)$.

The configuration is \#q $\mathrm{q}_{\mathrm{ret}}<\overline{\mathrm{ab} 32123}>\#$

- There is no more non overlined letter of I. So the automaton replaces the overlined letters by the same non overlined letters, verifying that all the data is overlined.

The configuration is \#<ab32123q reset $>$ \#.

- The head goes to the letter of I the most on the right. It is b.

The configuration is \#<aq again $\mathrm{b} 32123>\#$

- As before, the machine overlines $b$ and searches for the image of $b$ by $\psi$. It is 321 .

The configuration is \#<aqret $\overline{\text { b32123 }}>\#$

- The machine overlines a and searches for the image of a by $\psi$. It is 23 .

The configuration is now \# qret $<$ ab32123 $>\#$

- There is no more letter of I non overlined. So the automaton replaces the overlined letters by the same non overlined letters, verifying that all the tape is overlined.

The configuration is \#<ab32123 ? reset $>\#$

- Now, the machine has verified that the data had the form $\#<\tilde{m} m^{\prime}>\#$ with $\varphi(m)=\psi(m)=m$ '. $\tilde{m}$ represents the mirror of the word $m$.

- The initial configuration is restored when the Post correspondance problem $\mathrm{P}(\varphi, \psi)$ is satisfied. The configuration is \#<q0ab32123>\#.

\section{Example 2:}

The tape contains the word a132. The initial configuration is \#<q0a132>\#

- The machine overlines a. The configuration is \#<a $q_{\varphi(a) 1} 132>\#$

- It verifies that 1 is the first letter of $\varphi(a)$ and overlines it.

The configuration is now $\#<\overline{\mathrm{al}} \mathrm{q}_{\varphi(\mathrm{a}) 2} 32>\#$

- It verifies that 3 is the second letter of $\varphi(a)$. Since it is false, the machine stops.

Definition 1-4 : An initial configuration is proper if and only if it has the form $\#<q_{0} \tilde{m}_{I} m_{X}>\#$ (the mirror image of $m_{I}$ is represented by $\widetilde{m}_{I}$ ). with $m_{X}$ word in $X^{*}, m_{I}$ word in $I^{+}$, $q_{0}$ initial state of the machine. 


\section{Lemma 1-1 :}

For all machines of the class $A_{\text {Post }}$, if the initial configuration is not proper then the machine stops.

Proof: Using the definition of the machine.

\section{Lemma 1-2:}

For all machines in $A_{\text {post }}$ starting from a proper initial configuration $\#<q_{0} \tilde{m}_{I} m_{X}>\#$, the machine loops passing by its initial configuration again if and only if $m_{X}=\varphi\left(m_{l}\right)=\psi\left(m_{l}\right)$.

Proof: From the definition of the machine.

We get as corollary of these lemmas the next proposition.

\section{Proposition 1-2:}

(1) Termination in undecidable for the class $A_{p o s t}$

(2) If an automaton A $\varphi \psi$ loops for a data d then it passes by its initial configuration again.

Proof:

(1) According to lemmas 1-1 and 1-2, A $\varphi \psi$ loops if and only if $m_{X}=\varphi\left(m_{I}\right)=\psi\left(m_{I}\right)$. But it is not decidable wether $\varphi\left(m_{1}\right)=\psi\left(m_{I}\right)$ (Post correspondance problem). Therefore, termination of the class $A_{\text {Post }}$ is undecidable.

(2) From lemma 1-2, if the machine loops then it passes by its initial configuration again.

\section{Lemma 1-3 :}

If there exists a computation which loops from some configuration (not necessarily reachable) then there exists a beginning of computation starting from a proper initial configuration which loops.

Proof: See appendix II.

\section{II - Termination of non-length-increasing string rewriting systems}

We want to prove that termination of non-length-increasing string rewriting systems is undecidable.

Definition 2-1: A non-length-increasing string rewriting system is a system where rules have the form $1 \rightarrow r$ with ||$\geq|r| .1$ and $r$ are words.

A particular class of non-length-increasing string rewriting systems is the class of lengthpreserving string rewriting systems.

Definition 2-2 : A length-preserving string rewriting system is a system where rules have the form $1 \rightarrow \mathrm{r}$ with $|1|=|\mathrm{r}| .1$ and $\mathrm{r}$ are words. 
We construct a class $R_{\text {Post }}$ of rewriting systems associated to the class $A_{\text {Post }}$ of machines studied before: $\mathrm{I}$ and $\mathrm{X}$ are the two alphabets considered in paragraph I. $\Sigma=\{\#,<,>\} \cup \mathrm{I} \cup \mathrm{X}$. We constuct I' and I" from I, X' and X" from $X$, and $\Sigma^{\prime}, \Sigma$ " from $\Sigma: \forall a \in \Sigma, a^{\prime} \in \Sigma$ ' et a" $\in \Sigma^{\prime \prime}$. $Q$ is a finite alphabet, disjoint from $\Sigma$.

From a machine A $\varphi \psi$ of the class $A_{\text {post }}$, we construct the rewriting system R $\varphi \psi$.

For all transitions $\left(\mathrm{q}_{1}, \mathrm{a}_{1}\right) \rightarrow_{\mathrm{A}}\left(\mathrm{a}_{2}, \mathrm{q}_{2}, \mathrm{Ri}\right)$, for all $\mathrm{a}, \mathrm{b}$ in $\Sigma$, we construct the rule:

$$
a^{\prime} q_{1} a_{1} " b " \rightarrow \rightarrow_{R} a^{\prime} a_{2}^{\prime} q_{2} b^{\prime \prime}
$$

and for all transitions $\left(q_{1}, a_{1}\right) \rightarrow_{A}\left(a_{2}, q_{2}, L e\right)$, for all $a, b$ in $\Sigma$, we construct the rule:

$$
a^{\prime} b^{\prime} q_{1} a_{1} " \rightarrow_{R} a^{\prime} q_{2} b^{\prime \prime} a_{2} " \text {. }
$$

(intuitively, $\mathrm{x}^{\prime}$ is a letter on the left side of the tape head, and $\mathrm{x}$ " a letter on the right side.)

$R_{\text {Post }}$ is a class of length-preserving string rewriting systems.

Notation : to simplify, we write A for A $\varphi \psi$ and $R$ for the associated system R $\varphi \psi$.

$\rightarrow_{R}$ is the transitive reflexive closure of $\rightarrow_{R}$

\section{Lemma 2-1 :}

for all $\min \left(\Sigma^{\prime} \cup \Sigma^{\prime \prime} \cup Q\right)^{*}$,

- either $m \in\left(\Sigma^{\prime} \cup Q\right)^{*} .\left(\Sigma^{\prime \prime} \cup Q\right)^{*}$

- or $m$ can be written in one way $m_{1} a_{1}{ }^{\prime \prime} u_{1} b_{1}{ }^{\prime} m_{2} a_{2}{ }^{\prime \prime} u_{2} b_{2}{ }^{\prime} \ldots m_{n} a_{n}{ }^{\prime \prime} u_{n} b_{n}{ }^{\prime} m_{n+1}$ with $n>0$, $m_{1}, m_{2}, \ldots, m_{n+1} \in\left(\Sigma^{\prime} \cup Q\right)^{*} .\left(\Sigma^{\prime \prime} \cup Q\right)^{*}, u_{1}, u_{2}, \ldots, u_{n} \in Q^{*}, a_{1}{ }^{\prime}, \ldots, a_{n}^{\prime \prime} \in \Sigma^{\prime \prime}, b_{1}, \ldots, b_{n}{ }^{\prime} \in \Sigma^{\prime}$

Proof: By induction on the length of $\mathrm{m}$.

\section{Corollary 2-1:}

Every word $m$ of $\left(\Sigma^{\prime} \cup \Sigma^{\prime \prime} \cup Q\right)^{*}-\left(\Sigma^{\prime} \cup Q\right)^{*} .\left(\Sigma^{\prime \prime} \cup Q\right)^{*}$ can be written in one way $w_{1} w_{2} \ldots w_{n+1}$ with $w_{i} \in \Sigma^{\prime} .\left(\Sigma^{\prime} \cup Q\right)^{*}\left(\Sigma^{\prime \prime} \cup Q\right)^{*} . \Sigma^{\prime \prime}$ for $1<i<n+1$, and $w_{1}, w_{n+1} \in\left(\Sigma^{\prime} \cup Q\right)^{*} .\left(\Sigma^{\prime \prime} \cup Q\right)^{*}$.

\section{Proof:}

In the decomposition given by lemma 2-1, we suppose $w_{1}=m_{1} a_{1}$ " $u_{1}, w_{i}=b_{i}{ }^{\prime} m_{i+1} a_{i+1}$ " $u_{i+1}$ for $1<\mathrm{i}<\mathrm{n}+1$ and $\mathrm{w}_{\mathrm{n}+1}=\mathrm{b}_{\mathrm{n}}{ }^{\prime} \mathrm{m}_{\mathrm{n}+1}$

Definition 2-3 : Let $m$ be a word of $\left(\Sigma^{\prime} \cup \Sigma^{\prime \prime} \cup Q\right)^{*}$. We define the signature of $m$ by

- If $m \in\left(\Sigma^{\prime} \cup \mathrm{Q}\right)^{*} .\left(\Sigma^{\prime \prime} \cup \mathrm{Q}\right)^{*}$ then its signature is the empty set.

- Else, $m$ can be written in one way $m_{1} a_{1}{ }^{\prime} u_{1} b_{1}{ }^{\prime} \ldots m_{n} a_{n}{ }^{\prime} u_{n} b_{n}{ }^{\prime} m_{n+1}$ and its signature is the set of occurences of letters $a_{i}$ ". An occurence is denoted by the length of the shorted prefix of $m$ containing this occurence.

We remark that the signature underlines the occurences of letters of $\Sigma$ ", the successor of which is in $\Sigma$, without consideration of the states of $\mathrm{Q}$.

\section{Examples:}

$\Sigma=\{a, b, c, d, e, f, g\}, m=$ acfgc. The occurence of the first letter $c$ in $m$ is 2 , the occurence of the 
second letter $\mathrm{c}$ in $\mathrm{m}$ is 5 .

$\Sigma=\{0,1\}, Q=\{q\}$

$\mathrm{m}=0$ '1'0'q0'q0"1'" $\cdot \operatorname{sign}(\mathrm{m})=\varnothing$

$\mathrm{m}=0$ '1'0"q0"q0'1'q0'0"1' $\operatorname{sign}(\mathrm{m})=\{5,11\}$.

\section{Lemma 2-2 :}

The size and the signature of $a$ word $m$ are unchanged when $m$ is reduced with rules of $R$.

\section{Proof :}

Let $m$ be a word of $\left(\Sigma^{\prime} \cup \Sigma^{\prime \prime} \cup Q\right)^{*}$.

1st case: $\mathrm{m} \in\left(\Sigma^{\prime} \cup \mathrm{Q}\right)^{*} \cdot\left(\Sigma^{\prime \prime} \cup \mathrm{Q}\right)^{*}$.

then sign $(m)=\varnothing$. We apply on $m$ a rule of $R$.

a) the rule has the form $a^{\prime} q_{1} a_{1}$ " $b$ " $\rightarrow_{R} a^{\prime} a_{2} ' q_{2} b$ ", a and b some letters of $\Sigma$. This rule can be applied only if $m$ is written $m_{1} a^{\prime} q_{1} a_{1}$ "b' $m_{2}$ with $m_{1} \in\left(\Sigma^{\prime} \cup Q\right)^{*}$ and $m_{2} \in\left(\Sigma^{\prime} \cup Q\right)^{*}$. Then, applying the rule, we get the word $m^{\prime}=m_{1} a^{\prime} a_{2}^{\prime} \mathrm{q}_{2} b^{\prime \prime} m_{2}$. Hence $|m|=\left|m^{\prime}\right|=\left|m_{1}\right|+\left|m_{2}\right|+4$. Moreover, $\mathrm{m}^{\prime} \in\left(\Sigma^{\prime} \cup \mathrm{Q}\right)^{*} \cdot\left(\Sigma^{\prime \prime} \cup \mathrm{Q}\right)^{*}$ therefore $\operatorname{sign}\left(\mathrm{m}^{\prime}\right)=\varnothing=\operatorname{sign}(\mathrm{m})$.

b) the rule has the form $a^{\prime} b^{\prime} q_{1} a_{1}$ " $\rightarrow R a^{\prime} q_{2} b^{\prime \prime} a_{2}$ ", $a$ and $b$ some letters of $\Sigma$. This rule can be applied only if $m$ is written $m_{1} a^{\prime} b^{\prime} q_{1} a_{1} " m_{2}$ with $m_{1} \in\left(\Sigma^{\prime} \cup Q\right)^{*}$ and $m_{2} \in\left(\Sigma^{\prime} \cup Q\right)^{*}$. Applying this rule, we get the word $m^{\prime}=m_{1} a^{\prime} q_{2} b^{\prime \prime} a_{2} " m_{2}$. Hence $|m|=\left|m^{\prime}\right|=\left|m_{1}\right|+\left|m_{2}\right|+4$. Moreover, $\mathrm{m}^{\prime} \in\left(\Sigma^{\prime} \cup \mathrm{Q}\right)^{*} .\left(\Sigma^{\prime \prime} \cup \mathrm{Q}\right)^{*}$ therefore $\operatorname{sign}\left(\mathrm{m}^{\prime}\right)=\varnothing=\operatorname{sign}(\mathrm{m})$.

2nd case: $\mathrm{m}=\mathrm{m}_{1} \mathrm{a}_{1}$ " $\mathrm{u}_{1} \mathrm{~b}_{1}$ '... $\mathrm{m}_{\mathrm{n}} \mathrm{a}_{\mathrm{n}}$ " $\mathrm{u}_{\mathrm{n}} \mathrm{b}_{\mathrm{n}} \mathrm{m}_{\mathrm{n}+1}$

We apply one rule of $\mathrm{R}$ to $\mathrm{m}$. A rule can be applied only on a subword of $\mathrm{m}$ in $\left(\Sigma^{\prime} \cup \mathrm{Q}\right)^{*} .\left(\Sigma^{\prime \prime} \cup\right.$ $Q)^{*}$. But we have just shown that for $m \in\left(\Sigma^{\prime} \cup Q\right)^{*} .\left(\Sigma^{\prime \prime} \cup Q\right)^{*}$, rewriting preserves signature and size. Hence this result is true for each $\mathrm{m}$ in $\left(\Sigma^{\prime} \cup \Sigma^{\prime \prime} \cup Q\right)^{*}$

\section{Lemma 2-3 :}

For every word $m$ in $\left(\Sigma^{\prime} \cup \Sigma^{\prime \prime} \cup Q\right)^{*}-\left(\Sigma^{\prime} \cup Q\right)^{*} .\left(\Sigma^{\prime \prime} \cup Q\right)^{*}$ written $w_{1} w_{2} \ldots w_{n+1}$ $\left(m \rightarrow \rightarrow_{R} t\right) \Leftrightarrow\left(t=t_{1} t_{2} \ldots t_{n+1}\right.$ such that $\left.w_{i} \rightarrow_{R} t_{i}, i=1, \ldots, n+1\right)$

\section{Proof:}

$\Rightarrow$ Let $m=w_{1} w_{2} \ldots w_{n+1}$. We have shown with lemma $2-2$ that rules of $R$ can be applied only on $w_{i}$. Hence $(m \rightarrow R t) \Rightarrow\left(t=t_{1} t_{2} \ldots t_{n+1}\right.$ such that $\left.w_{i} \rightarrow_{R} t_{i}, i=1, \ldots, n+1\right)$

$\Leftrightarrow)$ if $t=t_{1} t_{2} \ldots t_{n+1}$ with $w_{i} \rightarrow_{R} t_{i}(i=1, \ldots, n+1)$ then $w_{1} w_{2} \ldots w_{n+1} \rightarrow t_{R} t_{1} t_{2} \ldots t_{n+1}$.

Therefore $m \rightarrow_{R} t . \square$

\section{Definition 2-4 :}

Let $m$ a word of form a'v'qw"b" with $q \in Q, v^{\prime} \in \Sigma^{*}, w^{\prime \prime} \in \Sigma^{\prime *}, a^{\prime} \in \Sigma^{\prime}$ and $b^{\prime \prime} \in \Sigma$ ". We associate to $\mathrm{m}$ the configuration of the machine $A: C(m)=a v q w b$.

Remark: $\mathrm{a}$ and $\mathrm{b}$ are never read by the machine and mark the end of the tape (instead of \#).

\section{Lemma 2-4 :}

$m \in\left(\Sigma^{\prime+} . Q . \Sigma^{\prime \prime}\right), m \rightarrow_{R} m^{\prime} \Leftrightarrow C(m) \rightarrow_{A} C\left(m^{\prime}\right)$. 
Proof:

$\Rightarrow$ ) To the word $\mathrm{m}$, we associate the configuration $\mathrm{C}(\mathrm{m})$. The rules of $\mathrm{R}$ have the following two forms:

(ri) $a$ 'q $q_{1} a_{1} " b " \rightarrow_{R} a^{\prime} a_{2}{ }^{\prime} q_{2} b "$, for some $a$ and $b$ in $\Sigma$.

(r2) $a^{\prime} b^{\prime} q_{1} a_{1} " \rightarrow_{R} a^{\prime} q_{2} b^{\prime \prime} a_{2}$ ", for some $a$ and $b$ in $\Sigma$.

1st case : rule $(\mathrm{r} 1)$.

To apply (rl) on $\mathrm{m}, \mathrm{m}$ should be of form $\mathrm{v}^{\prime} \mathrm{a}^{\prime} \mathrm{q}_{1} \mathrm{a}_{1}$ "b"w", $\mathrm{v}^{\prime} \in \Sigma \Sigma^{*}, \mathrm{w}^{\prime \prime} \in \Sigma$ "** Then $\mathrm{C}(\mathrm{m})=$ $v q_{1} a_{1} b w$. We know that $m-(r 1) \rightarrow_{R} m^{\prime} \Leftrightarrow m^{\prime}=v^{\prime} a^{\prime} a_{2}{ }^{\prime} q_{2} b^{\prime \prime} w "$. So $C\left(m^{\prime}\right)=v_{a a_{2}} q_{2} b w$. But $(\mathrm{r} 1)$ has been constructed from transition $\left(\mathrm{q}_{1}, \mathrm{a}\right) \rightarrow_{\mathrm{A}}\left(\mathrm{a}_{2}, \mathrm{q}_{2}, \mathrm{D}\right)$. Hence $\mathrm{C}(\mathrm{m}) \rightarrow_{\mathrm{A}} \mathrm{C}\left(\mathrm{m}^{\prime}\right)$.

2nd case : rule ( $\mathrm{r} 2$ ).

To apply ( $\mathrm{r} 2$ ) on $\mathrm{m}, \mathrm{m}$ should be of form $\mathrm{v}^{\prime} \mathrm{a}^{\prime} \mathrm{b}^{\prime} \mathrm{q}_{1} \mathrm{a}_{1}$ " $w$ ", $\mathrm{v}^{\prime} \in \Sigma^{\prime *}$ and $\mathrm{w}^{\prime \prime} \in \Sigma$ "*. Then $\mathrm{C}(\mathrm{m})$ $=v^{2} q_{1} a_{1} w$. Since $m-(r 1) \rightarrow m_{R} m^{\prime} \Leftrightarrow m^{\prime}=v^{\prime} a^{\prime} q_{2} b^{\prime \prime} a_{2}$ " $w^{\prime \prime}, C\left(m^{\prime}\right)=v a q_{2} b_{2} w$. But $(r 2)$ has been constructed from transition $\left(\mathrm{q}_{1}, \mathrm{a}\right) \rightarrow_{A}\left(\mathrm{a}_{2}, \mathrm{q}_{2}, \mathrm{G}\right)$. Hence $\mathrm{C}(\mathrm{m}) \rightarrow_{A} \mathrm{C}\left(\mathrm{m}^{\prime}\right)$.

$\Leftrightarrow$ Suppose that $m$ and $m^{\prime}$ are such that $C(m) \rightarrow_{A} C\left(m^{\prime}\right)$. Transitions of $A$ are of the following two forms:

(t1) $\left(\mathrm{q}_{1}, \mathrm{a}_{1}\right) \rightarrow \mathrm{A}\left(\mathrm{a}_{2}, \mathrm{q}_{2}, \mathrm{D}\right)$.

(t2) $\left(q_{1}, a 1\right) \rightarrow A\left(a_{2}, q_{2}, G\right)$.

$\mathrm{C}(\mathrm{m})=\alpha \sigma \mathrm{q}_{1} \mathrm{a}_{1} \omega \beta$ with $\alpha, \beta \in \Sigma$ and $\sigma, \omega \in \Sigma^{*}$. On $\mathrm{C}(\mathrm{m})$, we can apply (t1) or (t2).

1st case: transition ( $t 1)$.

To every transition (tI) and for all $a, b$ in $\Sigma$ we associate the rule (r1) : a' $q_{1} a_{1}$ "b" $\rightarrow_{R} a^{\prime} a_{2}{ }^{\prime} q_{2} b^{\prime \prime}$. The word $m$ associated to configuration $C(m)$ is $\alpha$ ' $\sigma^{\prime} q_{1} a_{1}$ " $\omega$ " $\beta$ ". The word $m^{\prime}$ such that $C(m)$ $-(t 1) \rightarrow_{A} C\left(m^{\prime}\right)$ is $\alpha^{\prime} \sigma^{\prime} a_{2}{ }^{\prime} q_{2} \omega^{\prime \prime} \beta$ ". Therefore $m^{\prime}=v^{\prime} a^{\prime} a_{2}{ }^{\prime} q_{2} b^{\prime \prime} w$ " supposing $v^{\prime} a^{\prime}=\alpha$ ' $\sigma^{\prime}$ and $b " w "=w " \beta "$. Then $m=v^{\prime} a^{\prime} q_{1} a_{1}$ "b"w". Hence, $m \rightarrow_{R} m$ '.

2nd case : transition ( $(2)$.

To every transition ( $t 2$ ) and for all $a, b$ in $\Sigma$ we associate the rule ( 2 ) : a'b' $q_{1} a_{1}$ " $\rightarrow \rightarrow_{R} a^{\prime} q_{2} b^{\prime \prime} a_{2}$ ". The word $m$ associated to $C(m)$ is $\alpha^{\prime} \sigma^{\prime} q_{1} a_{1} " \omega " \beta "$ (as in first case). $m$ is written $v$ 'a' $q_{1} a_{1}$ "b" $w$ " and the word $m$ ' such that $C(m)-(t 2) \rightarrow_{A} C\left(m\right.$ ') is written $v ' q_{2} a$ " $a_{2}$ "b" $w$ ". Since $C(m)$ is a configuration, $v$ ' contains at least one letter. Therefore we can apply a rule ( 12 ) and $\mathrm{m} \rightarrow_{\mathrm{R}} \mathrm{m}$.

\section{Lemma 2-5 :}

The machine $A$ does not terminate $\Rightarrow$ The rewriting system $R$ does not terminate

Proof: Suppose that the machine A does not terminate, then starting from an initial configuration $C_{0}$ there exists an infinite computation $C_{0} \rightarrow_{A} C_{1} \rightarrow A$... But for every configuration $C_{i}$, there exists a word $m_{i}$ in $\Sigma^{++} \cdot Q \cdot \Sigma^{\prime+}$ such that $C_{i}=C\left(m_{i}\right)$. Hence there exists $\mathrm{m}_{0}, \mathrm{~m}_{1}, \ldots$ in $\Sigma^{\prime+} \cdot \mathrm{Q} \cdot \Sigma^{\prime \prime+}$ such that $\mathrm{C}\left(\mathrm{m}_{0}\right) \rightarrow_{\mathrm{A}} \mathrm{C}\left(\mathrm{m}_{1}\right) \rightarrow_{\mathrm{A}} \ldots$. According to lemma $2-4, \mathrm{C}\left(\mathrm{m}_{0}\right)$ $\rightarrow_{\mathrm{A}} \mathrm{C}\left(\mathrm{m}_{1}\right) \rightarrow_{\mathrm{A}} \ldots \Leftrightarrow \mathrm{m}_{0} \rightarrow_{\mathrm{R}} \mathrm{m}_{1} \rightarrow_{\mathrm{R}} \ldots$. Therefore $\mathrm{R}$ does not terminate.

\section{Lemma 2-6 :}

The rewriting system $R$ does not terminate $\Rightarrow$ there exists an infinite subcomputation $C_{1} \rightarrow{ }_{A} C_{2}$ $\rightarrow_{A} \cdots$

Proof: We suppose that $\mathrm{R}$ does not terminate.

Hence there exists an infinite derivation $m \rightarrow_{R} \ldots$. From lemma $2-1$, either $m \in(\Sigma, \cup Q)^{*} .(\Sigma$ " 
$\cup Q)^{*}$, or $m$ is written $m_{1} a_{1} " u_{1} b_{1}{ }^{\prime} \ldots m_{n+1}$.

1) if $m \in\left(\Sigma^{\prime} \cup Q\right)^{*} .\left(\Sigma^{\prime \prime} \cup Q\right)^{*}$

On $m$, we can apply a rule of $R$. Therefore $m=v_{1} \sigma v_{2}$ with $v_{1} \in\left(\Sigma^{\prime} \cup Q^{*} \cdot Q^{+}\right.$or $v_{1} \in Q^{*}, v_{2}$ $\in \mathrm{Q}^{+} .\left(\Sigma^{\prime \prime} \cup \mathrm{Q}\right)^{*}$ or $v_{2} \in \mathrm{Q}^{*}$, and $\sigma \in \Sigma^{\prime+} . \mathrm{Q} . \Sigma^{\prime \prime}$. To $\sigma$ we associate the $\mathrm{A}$ configuration : $\mathrm{C}(\sigma)$. We cannot apply a rule on $v_{1}$ and $v_{2}$, so we apply it on $\sigma$. Hence $\sigma \rightarrow_{R} \ldots$. According to lemma $2-4, \mathrm{C}(\sigma) \rightarrow \mathrm{A} \cdots$

2) else $m=m_{1} a_{1} " u_{1} b_{1}{ }^{\prime} m_{2} a_{2} " u_{2} b_{2}{ }^{\prime} \ldots m_{n+1}=w_{1} w_{2} \ldots w_{n+1}(l e m m a ~ 2-2)$.

From lemma 2-3, $m \rightarrow R t \Leftrightarrow t=t_{1} \ldots t_{n+1}$ with $w_{i} \rightarrow t_{R} t_{i}$. Therefore, if the rewriting is infinite, there exists a $w_{i}$ such that rewriting restricted to $w_{i}$ is infinite. But we know that $w_{i} \in\left(\Sigma^{\prime} \cup Q\right)^{*} .\left(\Sigma^{\prime \prime}\right.$ $\cup$ Q)*, so we can use the precedent case. $\square$

Theorem 2-1: Termination of length-preserving string rewriting systems is undecidable.

Proof: It suffices to verify that construction which associates a string rewriting system $R \varphi \psi$ to the linear-bounded automaton $\mathrm{A} \varphi \psi$ reduces termination problem in class $A_{\text {Post }}$ to termination problem for length-preserving string rewriting systems. For that, we can remark that proposition 1-2, lemma 1-3 and lemma 2-6 involve that if R $\varphi \psi$ does not terminate neither do A $\varphi \psi$. Indeed, if $R \varphi \psi$ does not terminate, $A \varphi \psi$ passes by a proper initial configuration again from which it loops.

From Lemma 2-5 we obtain the converse of the theorem. $\square$

\section{Corollary 2-2 :}

Termination of non-length-increasing string rewriting system is undecidable.

Remark :

$\mathrm{A} \varphi \psi$ could loop from a non-reachable configuration and stopped from every initial configurations. Therefore it could terminate in the machine sense, without assure termination for R $\varphi \psi$. Lemma 1-3 avoids this problem.

\section{III - Undecidability of confluence of terminating rewriting systems on q $\Lambda^{*}$}

In this section, we want to show that properties for linear-bounded automata cannot always be translated for non-length-increasing string rewriting systems. Indeed, for a linear-bounded automaton we start from an initial configuration, where the tape head is on the left side of the tape. That should mean, for a rewriting system, that we work on words starting by a special letter symbolizing initial state of linear-bounded automaton.

Definition 3-1 : [Huet]

A rewriting system is confluent if for all $u$ that reduces to two terms $t$ and $t^{\prime}$ there exists $v$ such that $t$ and $t^{\prime}$ reduce to $v$. 
Theorem 3-1 : [Newman]

Confluence is decidable for terminating rewriting systems.

It is well known that confluence and ground confluence (confluence restricted to terms without variables) are not equivalent. In particular, ground confluence is undecidable for terminating term rewriting systems. Confluence implies ground confluence but the converse is false. Nevertheless, the following identification lemma shows that we can identify confluence of any semi-Thue system $\mathrm{S}$ with both confluence and ground confluence of the corresponding term rewriting system $S^{\prime}$.

\section{Identification lemma :}

Let $S$ be a semi-Thue system over a (non ranked) alphabet $\Sigma$. We associate to $S$ the term rewriting system $S^{\prime}$ over the ranked alphabet $\Sigma^{\prime}=\{a(x) / x \in \Sigma\} \cup\{\$\}$. (\$is a constant).

$S^{\prime}=\{l(x) \rightarrow r(x) / l \rightarrow r \in S\}$

Then $t \rightarrow S u \Leftrightarrow t(x) \rightarrow S^{\prime} u(x) \Leftrightarrow t(\$) \rightarrow S^{\prime} u(\$)$

proof : obvious

As a corollary, confluence of $S$, confluence and ground confluence of S' coincide.

From now, we use this identification and work only in the word case. We describe in this paragraph a terminating rewriting system. This system is not confluent on words of $\Lambda^{*}$ and its confluence is undecidable on $\mathrm{q} \Lambda^{*}, \mathrm{q}$ a fixed symbol of $\Lambda$.

Let A $\varphi \psi$ be a machine of class $A_{P o s t}$ studied in part I. We associate to this machine an alphabet: $\Lambda=\mathrm{Q} \varphi \Psi \cup \Sigma \cup\left\{\mathrm{q}, \mathrm{q}_{\mathrm{yes}}, \mathrm{X}, \mathrm{N}\right\}$. We modify the machine A$\varphi \psi$. We remote the last transitions which make the machine loop. Therefore, if the data has the form $\tilde{\mathrm{mm}}{ }^{\prime}$ with $\mathrm{m}^{\prime}=\varphi(\mathrm{m})=\psi(\mathrm{m})$, the machine goes to the configuration \#q $q_{\text {end }}<\tilde{n} m$ ' $>\#$ and stops.

To this new automaton, we associate a rewrite system $R 1$. It contains all the rules simulating the transitions of the machine: A transition of form $(\mathrm{q}, \mathrm{a}) \rightarrow_{\mathrm{A} \varphi \mathrm{Y}}\left(\mathrm{b}, \mathrm{q}^{\prime}, \mathrm{Le}\right)$ is associated to the rule $\mathrm{hqa} \rightarrow \mathrm{R} 1 \mathrm{q}^{\prime} \mathrm{hb}, \mathrm{a}, \mathrm{b}$, $\mathrm{h}$ in $\Lambda$ and $\mathrm{q}, \mathrm{q}^{\prime}$ in $\mathrm{Q} \varphi \psi$; A transition of form $(\mathrm{q}, \mathrm{a}) \rightarrow_{\mathrm{A} \varphi \varphi}\left(\mathrm{b}, \mathrm{q}^{\prime}, \mathrm{Ri}\right)$ is associated to the rule qa $\rightarrow_{\mathrm{R} 1} \mathrm{bq}, \mathrm{a}, \mathrm{b}$, in $\Lambda$ and $\mathrm{q}$, q' in $\mathrm{Q} \varphi \psi$.

Moreover, R1 contains the rules:

$\mathrm{q}_{\text {end }}<\rightarrow \mathrm{R} 1$ qyes

$\forall \mathrm{a} \in \Lambda-\{\#\} \mathrm{q}_{\text {yes }} \mathrm{a} \rightarrow \mathrm{R} 1$ qyes

qyes $^{\# \rightarrow} \rightarrow \rightarrow_{\mathrm{R} 1} \mathrm{Y}$

$\mathrm{q} \rightarrow \mathrm{R}_{\mathrm{R}}<\mathrm{q}_{\mathrm{O}}$

We consider now this rewriting system $\mathrm{R} 2$ :

$\forall \mathrm{f} \in \Lambda-\{\mathrm{Y}, \mathrm{N}\}, \mathrm{f} \rightarrow{ }_{\mathrm{R} 2} \mathrm{~N}$

$\forall \mathrm{a} \in \Lambda, \mathrm{Ya} \rightarrow_{\mathrm{R} 2} \mathrm{~N}$

$\forall \mathrm{a} \in \Lambda, \mathrm{Na} \rightarrow{ }_{\mathrm{R} 2} \mathrm{~N}$

$R$ is the system constituted by the rules of $R 1$ and $R 2$. $R$ is terminating.

Lemma 3-1 :

$m \in I^{+}, m^{\prime} \in X^{*}, q \tilde{m} m^{\prime}>\# \rightarrow_{R} Y$ if and only if $\varphi(m)=\psi(m)=m^{\prime}$. 
(we denote $\tilde{m}$ the mirror image of $m$ )

\section{Proof:}

$\Leftarrow)$ if $\varphi(m)=\psi(m)=m^{\prime}$ then according to part $I$, the machine $A \varphi \psi$, from an initial configuration $\#<q_{0} i_{n} \ldots i_{1} x_{1} \ldots x_{p}>\#$ goes to the instantaneous description $\# q_{\text {end }}<i_{n} \ldots i_{1} x_{1} \ldots x_{p}>\#$ with $m=i_{1} \ldots i_{n}$ and $m^{\prime}=x_{1} \ldots x_{p}$. Hence with $R$ we can go from the word $q i_{n} \ldots i_{1} x_{1} \ldots x_{p}>\#$ to the word $<q_{0} i_{n} \ldots i_{1} x_{1} \ldots x_{p}>\#$ and to $q_{\text {end }}<i_{n} \ldots i_{1} x_{1} \ldots x_{p}>\#$. Applying the rule $q_{e n d}<\rightarrow \rightarrow_{R 1} q_{y e s}$ we get $q_{y e s} i_{11} \ldots i_{1} x_{1} \ldots x_{p}>\#$. Applying several times rules of form $q_{y e s} \rightarrow_{R 1} q_{y e s}$ we get the word qyes $\#$. Finally with the rule qyes $\# \rightarrow_{R 1} Y$ we get the word $Y$.

$\Rightarrow$ ) we suppose that qûmm' $>\# \rightarrow_{R} Y$ with $m=i_{1} \ldots i_{n}$ and $m^{\prime}=x_{1} \ldots x_{p}$.

$m$ and $m$ ' do not contain $Y$ neither qyes neither any state of $Q \varphi \psi$. There is only one manner to get $Y$ : using the rule $q_{y e s} \# \rightarrow_{R 1}$ Y. Moreover, the only manner to get $q_{y e s}$ is to apply the rule $q_{\text {end }}<\rightarrow \rightarrow_{\mathrm{R} 1} q_{\text {yes. }}$. To generate $q_{\text {end }}$, we have applied rules simulating $A \varphi \psi . q i_{1} \ldots i_{1} x_{1} \ldots x_{p}>\# \rightarrow R$ $<q_{0} i_{n} \ldots i_{1} x_{1} \ldots x_{p}>\# \rightarrow \rightarrow_{R} q_{e n d}<i_{n} \ldots i_{1} x_{1} \ldots x_{p}>\#$.

But, we have seen in paragraph $I$ that $\#\left\langle q_{0} i_{n} \ldots i_{1} x_{1} \ldots x_{p}>\# \rightarrow A \rightarrow_{A} \# q_{\text {end }}<i_{n} \ldots i_{1} x_{1} \ldots x_{p}>\#\right.$ if and only if $\varphi\left(i_{1} \ldots i_{11}\right)=\psi\left(i_{1} \ldots i_{n}\right)=x_{1} \ldots x_{p}$.

\section{Definition 3-2:}

A word $w$ is a normal form if there exists no word $v$ with $w \rightarrow_{R} v$.

A word $w$ has a normal form if $w \rightarrow{ }_{R} v$ for some normal form $v$.

The set of normal forms of a word $m$ (called irreducible forms) is denoted by IRR(m).

\section{Lemma 3-2 :}

For all $u$ in $\Lambda^{+}-\{Y\}, N$ is in $\operatorname{IRR}(u)$.

\section{Proof:}

- if $\mathrm{u}$ does not start by $\mathrm{N}$ neither by $\mathrm{Y}$. Then applying the rule $\mathrm{f} \rightarrow_{\mathrm{R} 2} \mathrm{~N}$ and possibly the rules of form $\mathrm{Na} \rightarrow_{\mathrm{R} 2} \mathrm{~N}$ we obtain the reduction $\mathrm{u} \rightarrow_{\mathrm{R}} \mathrm{N}$. So $\mathrm{N}$ is in $\operatorname{IRR}(\mathrm{u})$.

- if $u$ starts by $N: u=N . w$

If $w=\varepsilon$ then $u=N$ and so $u$ is irreducible. Hence $N$ is in IRR(u). Else we apply several times rules $\mathrm{Na} \rightarrow{ }_{\mathrm{R} 2} \mathrm{~N}$ and we get $\mathrm{N}$ in IRR(u).

- if $u$ starts by $Y: u=Y$. $w$ with $w \neq \varepsilon$. Hence we can apply a rule $\mathrm{Ya} \rightarrow \rightarrow_{R 2} N$ and possibly rules $\mathrm{Na} \rightarrow{ }_{R 2} \mathrm{~N}$. Therefore $\mathrm{N}$ is in IRR(u).

\section{Lemma 3-3 :}

$\forall m \in \Lambda^{*}, \operatorname{IRR}(m) \subset\{Y, N, \varepsilon\} . \forall m \in \Lambda^{+}, \operatorname{IRR}(m) \subset\{X, N\}$

\section{Proof:}

Suppose that there exists $\mathrm{u} \in \operatorname{IRR}(\mathrm{m}), \mathrm{u} \notin\{\mathrm{Y}, \mathrm{N}, \varepsilon\}$. Then, from lemma $3-2$, $\mathrm{u}$ can be reduced to $N$. So $u$ is not a normal form. Moreover, it is obvious that $\varepsilon \in \operatorname{IRR}(m)$ if and only if $m=\varepsilon$.

\section{Lemma 3-4 :}

Let $q w$ be a word in $q \Lambda^{*}$.

If qw has the form qm̃m'>

i) $\operatorname{IRR}(q w)=\{Y, N\}$ iff $\varphi(m)=\psi(m)=m^{\prime}$ 
ii) $I R R(q w)=\{N\}$ iff $\varphi(m) \neq m^{\prime}$ or $\psi(m) \neq m^{\prime}$

Else IRR $(q w)=\{N\}$.

Proof:

1) qw has the form qũm'>\#

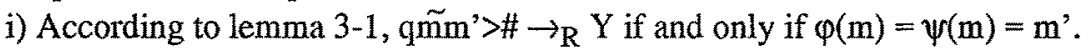

Moreover qũm' $>\# \rightarrow_{R} N$ from lemma 3-2. Hence $\{Y, N\} \subset I R R(q w)$ if and only if $\varphi(m)=$ $\psi(m)=m^{\prime}$. Lemma 3-3 shows that IRR $(q w)=\{Y, N\}$ iff $\varphi(m)=\psi(m)=m^{\prime}$.

ii) $\varphi(m) \neq m^{\prime}$ or $\psi(m) \neq m^{\prime} \Leftrightarrow Y \notin \operatorname{IRR}(q w)$. But qũm' $>\# \rightarrow_{R} N$. Hence $\operatorname{IRR}(q w)=\{N\}$

2) qw has not the form qũm' $>\#$. Then qw does not correspond to a proper initial configuration of A $\varphi \psi$. Hence we apply rules of $R$ without obtain the word $\mathrm{q}_{\text {end }}<\mathrm{m}$. Therefore $\mathrm{Y} \notin \mathbb{R} R(\mathrm{qw})$. But $q w \rightarrow_{R} N$. Hence IRR $(q w)=\{N\}$.

Theorem 3.2: $q$ is a fixed symbol of a finite alphabet $\Lambda$. The following problem is undecidable. Instance : R semi-Thue, non-length-increasing, terminating, not confluent.

Question : is $R$ confluent on $q \Lambda^{*}$ ?

Proof: According to lemma 3-4, $\mathrm{R}$ is divergent on $\mathrm{q} \Lambda^{*}$ if and only if there exists $\mathrm{m}$ such that $\varphi(m)=\psi(m)$. Hence R convergence on $q \Lambda^{*}$ is equivalent to Post problem. Consequently it's undecidable.

Using the identification lemma, we get the corollary:

Corollary 3-1: The following problem is undecidable.

Instance: $R$ a terminating, rewriting system. S a sort.

Question : is $R$ confluent on $S$ ?

Remark: $\mathrm{S}$ can be choosen very simple. For example, $\mathrm{S}$ is the set of terms of root $\mathrm{q}$.

\section{References}

[Book] Personnal communication.

[Dauchet] Max Dauchet

"Simulation of Turing Machines by a left-linear rewrite rule"

Rewriting Techniques and Applications. 3rd international conference, RTA-89

Chapel Hill, North Carolina, USA, April 1989 Proceedings in LNCS 355

N. Dershowitz (Ed.) p 109-120 (1987)

[Dershowitz] Nachum Dershowitz

"Termination of Rewriting" J.Symbolic Computation (1987) 3, 69-116

[Dershowitz \& Jouannaud] N. Dershowitz and J.P. Jouannaud

"Rewrite systems" Rapport de recherche 478. Unité associée au CNRS 410. (1989) 
[Hooper] Philip K. Hooper

"The undecidability of the Turing machine immortality problem"

J.Symbolic Logic 31 (2) June 1966. (1966)

[Hopcroft \& Ullman] J.E. Hopcroft and J.D. Ullman

"Some results on tape-bounded Turing machines"

J.A.C.M. Vol 16 (1), January 1967, pp 168-177.

[Huet] Gérard Huet

"Confluent reductions: abstact ptoperties and applications to term rewriting systems"

J.A.C.M. Vol 27, (4), October 1980 pp 797-821. (1980)

[Huet \& Lankford] G. Huet and D.S. Lankford

"On the uniform halting problem for term rewriting systems", Rapport laboria 283, Institut de Recherche en Informatique et en automatique, Le Chesnay, France, Mars 1978. (1978)

[Kuroda] S.-Y. Kuroda

"Classes of languages and linear-bounded automata"

Information and Controle 7, 207-223 (1964).

[Litovsky \& Metivier] Igor Litovsky and Yves Metivier

"Computing with graph rewriting systems with priorities" Rapport interne LaBRI 90-87

[Myhill] J.Myhill

"Linear bounded automata"

WADD Tech. Note No. 60-165, Wright-Patterson Air Force Base, Ohio. (1960)

[Newman] M.H.A. Newman

"On theories with a combinatorial definition of equivalence"

Annals of Mathematics 43 (2), p. 223-243. (1942)

[Otto] Friedrich Otto

"On deciding the confluence of a finite string-rewriting system on a given congruence class"

J. Comput. System Sciences 35, 285-310 (1987)

[Post] Emil L. Post

"A variant of a recursively unsolvable problem".

Bulletin of the American Mathematical Society 52 p 264-268. (1946)

\section{Appendix I : Definition of a machine A $\varphi \Psi$}

In the transitions, for $i$ in $I, \varphi(i)_{j}$ represents $j^{\text {rd }}$ letter of $\varphi(i)$, $\varphi(\mathrm{i})=\varphi(\mathrm{i})_{1} \ldots \varphi(\mathrm{i})_{\mathrm{ki}}$. We use the same notation for $\psi: \psi(\mathrm{i})_{\mathbf{j}}$ represents $\mathrm{j}^{\text {rd }}$ letter of $\psi(\mathrm{i}), \psi(\mathrm{i})=$ 
$\psi(\mathrm{i})_{1} \ldots \psi(\mathrm{i})_{\mathrm{ri}}$.

$\mathrm{Q} \varphi \psi=\left\{\mathrm{q}_{0}, \mathrm{q}_{1}, \mathrm{q}_{2}, \mathrm{q}_{\mathrm{ret}}, \mathrm{q}_{\text {reset }}, \mathrm{q}_{\mathrm{again}}, \mathrm{q}_{\mathrm{ret}}, \mathrm{q}_{\text {reset }}, \mathrm{q}_{\text {end }}\right\} \cup\left\{\mathrm{q}_{\varphi(\mathrm{i}) \mathrm{j}} / \mathrm{i} \in \mathrm{I}\right.$ and $\varphi(\mathrm{i})=\varphi(\mathrm{i})_{1} \ldots \varphi(\mathrm{i})_{\mathrm{k}_{\mathrm{i}}}$, $\mathrm{j} \in\left[1, \mathrm{k}_{\mathrm{j}}\right]$ if $\left.\varphi(\mathrm{i}) \neq \varepsilon\right\} \cup\left\{\mathrm{q}_{\varphi(\mathrm{i}) 0}\right.$ if $\left.\varphi(\mathrm{i})=\varepsilon\right\} \cup\left\{\mathrm{q}_{\psi(\mathrm{i}) \mathrm{j}} / \mathrm{i} \in \mathrm{I}\right.$ and $\psi(\mathrm{i})=\psi(\mathrm{i})_{1} \ldots \psi(\mathrm{i})_{\mathrm{r}_{\mathrm{i}}}, \mathrm{j} \in\left[1, \mathrm{r}_{\mathrm{i}}\right]$ if $\psi(\mathrm{i}) \neq \varepsilon\} \cup\left\{q_{\psi(i) 0}\right.$ if $\left.\psi(\mathrm{i})=\varepsilon\right\}$.

. The tape is of form \#<m>\#, $m$ is a word in $(I \cup X)^{*}$. initially, tape head is on the first letter of $\mathrm{m}$, and machine state is $\mathrm{q}_{0}$.

$\Delta$ is the next-move function:

A transition $(q, a) \rightarrow\left(b, q^{3}\right.$, Dir $)$ means that the head reads the letter $a$, the state of the automaton is $q$, and after applying the transition, the letter replacing $a$ is b, the new state is q' and the head goes to the direction Dir (Ri for rigth, Le for left).

$\forall \mathrm{i}, \mathrm{i}^{\prime} \in \mathrm{I}$ and $\forall \mathrm{x} \in \mathrm{X}$,

a) rules of equality verification: $m_{X}=\varphi\left(m_{I}\right)$.

$\left(\mathrm{q}_{0}, \mathrm{i}\right) \rightarrow\left(\mathrm{i}, \mathrm{q}_{1}, \mathrm{Ri}\right)$

$\%$ research of $i$ on the rigth side

$\left(\mathrm{q}_{1}, \mathrm{i}\right) \rightarrow\left(\mathrm{i}, \mathrm{q}_{1}, \mathrm{Ri}\right)$

$\left(\mathrm{q}_{1}, \mathrm{x}\right) \rightarrow\left(\mathrm{x}, \mathrm{q}_{2}, \mathrm{Le}\right)$

$\left(\mathrm{q}_{2}, \mathrm{i}\right) \rightarrow\left(\overline{\mathrm{i}}, \mathrm{q}_{\mathrm{p}(\mathrm{i}) 1}, \mathrm{Ri}\right)$

$\%$ research of its image by $\varphi$ if it is not $\varepsilon$.

$\left(\mathrm{q}_{\varphi(\mathrm{i}) 1}, \overline{\mathrm{i}}\right) \rightarrow\left(\overline{\mathrm{i}}, \mathrm{q}_{\varphi(\mathrm{i}) 1}, \mathrm{Ri}\right)$

$\left(\mathrm{q}_{\varphi(\mathrm{i}) 1}, \overline{\mathrm{x}}\right) \rightarrow\left(\overline{\mathrm{x}}, \mathrm{q}_{\varphi(\mathrm{i}) 1}, \mathrm{Ri}\right)$

$\left(\mathrm{q}_{\varphi(\mathrm{i}) \mathrm{j}}, \varphi(\mathrm{i})_{\mathrm{j}}\right) \rightarrow\left(\overline{\varphi(\mathrm{i})_{\mathrm{j}}}, \mathrm{q}_{\varphi(\mathrm{i}) \mathrm{j}+1}, \mathrm{Ri}\right)$

$\left(\mathrm{q}_{\varphi(\mathrm{i}) \mathrm{ki}_{\mathrm{i}}}, \varphi(\mathrm{i})_{\mathrm{kj}_{\mathrm{j}}}\right) \rightarrow\left(\overline{(\varphi(\mathrm{i})}_{\mathrm{ki}_{\mathrm{i}}}, \mathrm{q}_{\mathrm{ret}}, \mathrm{Le}\right) \quad \%$ we do it again for next $\mathrm{i}$.

$\left(q_{\text {ret }}, \bar{x}\right) \rightarrow\left(\bar{x}, q_{\text {ret }}\right.$, Le $)$

$\left(\mathrm{q}_{\text {ret }}, \overline{\mathrm{i}}\right) \rightarrow\left(\overline{\mathrm{i}}, \mathrm{q}_{\text {ret }}, \mathrm{Le}\right)$

$\left(\mathrm{q}_{\text {ret }}, \mathrm{i}\right) \rightarrow\left(\overline{\mathrm{i}}, \mathrm{q}_{\varphi(\mathrm{i}) 1}, \mathrm{Ri}\right)$

$\%$ if the image of $i$ by $\varphi$ is not $\varepsilon$

$\left(q_{\text {ret }}, i\right) \rightarrow\left(\vec{i}, q_{\varphi(i) 0}\right.$, Le $)$

$\%$ if the image of $i$ by $\varphi$ is $\varepsilon$

$\left(q_{\text {ret }},<\right) \rightarrow\left(<, q_{\text {reset }}, \mathrm{Ri}\right)$

$\%$ if the image of $i$ by $\varphi$ is $\varepsilon$

$\left(q_{2}, \mathrm{i}\right) \rightarrow\left(\overline{\mathrm{i}}, \mathrm{q}_{\varphi(\mathrm{i}) 0}, \mathrm{Le}\right)$

$\%$ if the image of i' by $\varphi$ is not $\varepsilon$

$\left(\mathrm{q}_{\varphi(\mathrm{i}) 0}, \mathrm{i}^{\prime}\right) \rightarrow\left(\mathrm{i}^{3}, \mathrm{q}_{\varphi\left(\mathrm{i}^{\prime}\right) 1}, \mathrm{Ri}\right)$

$\%$ if the image of $i$ ' by $\varphi$ is $\varepsilon$

$\left.\left(\mathrm{q}_{\text {(i) }} \mathrm{i}\right),<\right) \rightarrow\left(<, \mathrm{q}_{\text {reset }}, \mathrm{Ri}\right)$

b) rules of tape reset.

$\left(\mathrm{q}_{\text {reset }}, \overline{\mathrm{i}}\right) \rightarrow\left(\mathrm{i}, \mathrm{q}_{\text {reset }}, \mathrm{Ri}\right)$

(qreset, $\bar{x}) \rightarrow\left(x, q_{\text {reset }}, R i\right)$

$\left(q_{\text {reset }},>\right) \rightarrow(>$, qrecom, Le $)$

$\left(q_{\text {again }}, x\right) \rightarrow\left(x, q_{\text {again }}, L e\right)$

c) rules of equality verification: $m_{X}=\psi\left(m_{I}\right)$.
$\left(\mathrm{q}_{\text {again }}, \mathrm{i}\right) \rightarrow\left(\overline{\mathrm{i}}, \mathrm{q}_{\psi(\mathrm{i}) 1}, \mathrm{Ri}\right)$
$\%$ if the image of $\mathrm{i}$ by $\psi$ is not $\varepsilon$
$\left(\mathrm{q}_{\psi(\mathrm{i}) 1}, \overline{\mathrm{i}}\right) \rightarrow\left(\overline{\mathrm{i}}, \mathrm{q}_{\psi(\mathrm{i}) 1}, \mathrm{Ri}\right)$
$\left(\mathrm{q}_{\psi(\mathrm{i}) 1}, \overline{\mathrm{x}}\right) \rightarrow\left(\overline{\mathrm{x}}, \mathrm{q}_{y(\mathrm{i}) 1}, \mathrm{Ri}\right)$
$\left(q_{\psi(i) j}, \psi(i)_{j}\right) \rightarrow\left(\overline{\psi(i)}, q_{\psi(i) j+1}, R i\right)$
$\left(\mathrm{q}_{\psi(\mathrm{i}) \mathrm{ri}}, \psi(\mathrm{i})_{\mathrm{ri}}\right) \rightarrow\left(\overline{\psi(\mathrm{i})_{\mathrm{ri}}}, \mathrm{q}_{\mathrm{ret}}^{\prime}, \mathrm{Le}\right)$
$\left(q_{\text {ret }}^{\prime}, \bar{x}\right) \rightarrow\left(\bar{x}, q_{\text {ret }}^{\prime}\right.$, Le $)$
$\left(\mathrm{q}_{\text {ret }}^{\prime}, \overline{\mathrm{i}}\right) \rightarrow\left(\overline{\mathrm{i}}, \mathrm{q}_{\mathrm{ret}}^{\text {set }}, \mathrm{Le}\right)$ 
$\left(\mathrm{q}_{\text {ret }}^{\prime}, \mathrm{i}\right) \rightarrow\left(\overline{\mathrm{i}}, \mathrm{q}_{\psi(\mathrm{i}) 1}, \mathrm{Ri}\right)$

$\left(\mathrm{q}_{\text {ret }}^{\prime}, \mathrm{i}\right) \rightarrow\left(\mathrm{i}, \mathrm{q}_{y}(\mathrm{i}) 0, \mathrm{Le}\right)$

$\left(q_{\text {ret }},<\right) \rightarrow\left(<, q_{\text {reset }}, R i\right)$

$\left(\mathrm{q}_{\text {again }}, \mathrm{i}\right) \rightarrow\left(\overline{\mathrm{i}}, \mathrm{q}_{\psi(\mathrm{i}) 0}, \mathrm{Le}\right)$

$\%$ if the image of $i$ by $\psi$ is $\varepsilon$

$\left(\mathrm{q}_{\psi(\mathrm{i}) 0}, \mathrm{i}^{\prime}\right) \rightarrow\left(\overline{\mathrm{i}^{\prime}}, \mathrm{q}_{\psi\left(\mathrm{i}^{\prime}\right) 1}, \mathrm{Ri}\right)$

$\left(q_{\psi(i) 0}, i^{\prime}\right) \rightarrow\left(\bar{i}^{\prime}, q_{\psi(i ') 0}, L e\right)$

$\left(\mathrm{q}_{\psi(\mathrm{i}) 0},<\right) \rightarrow\left(<, \mathrm{q}_{\text {reset }}^{\mathrm{B}}, \mathrm{Ri}\right)$

d) rules of restoration of initial configuration

$\left(q_{\text {reset }}, \overline{\mathrm{i}}\right) \rightarrow\left(\mathrm{i}, \mathrm{q}_{\text {reset }}^{\prime}, \mathrm{Ri}\right)$

$\left(\mathfrak{q}_{\text {reset }}, \bar{x}\right) \rightarrow\left(x\right.$, qreset $\left._{\text {ret }}, \mathrm{Ri}\right)$

(q $\left.q_{\text {reset }},>\right) \rightarrow\left(>, q_{\text {end }}\right.$, Le $)$

$\left(\mathrm{q}_{\text {end }}, \mathrm{x}\right) \rightarrow\left(\mathrm{x}, \mathrm{q}_{\mathrm{end}}, \mathrm{Le}\right)$

(qend , i) $\rightarrow\left(\mathrm{i}, \mathrm{q}_{\text {end }}, \mathrm{Le}\right)$

( $\left.\mathrm{q}_{\text {end }},<\right) \rightarrow\left(<, \mathrm{q}_{0}, \mathrm{Ri}\right)$

\section{Appendix II : Proof of lemma 1-3}

Let ID a configuration such that A $\varphi \psi$ loops from it.

First case: ID is reachable

If ID is reachable, there exists an initial configuration from which the machine has reached ID. Therefore, there exists a beginning of computation which loops. The machine A $\varphi \psi$ loops if andonly if $\mathrm{P}(\varphi, \psi)$ is verified. Moreover, when Post property is verified, the machine loops passing by a proper initial configuration again. Hence there exists a beginning of computation starting from a proper initial configuration which loops.

Second case: ID is not reachable.

Let's see the dependence graph between states of $A \varphi \psi$.

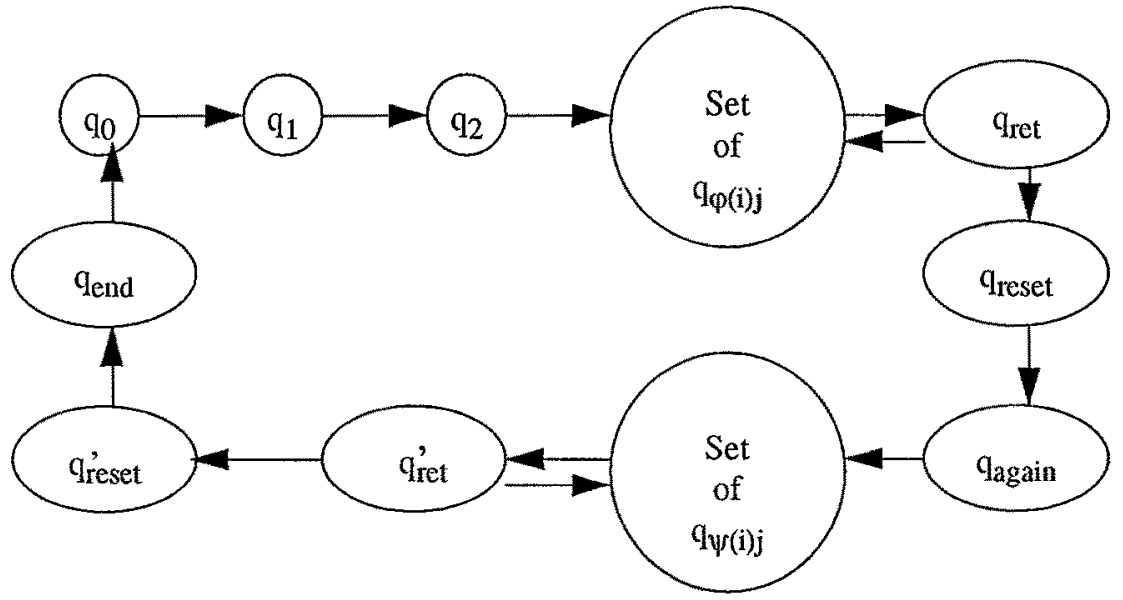

We write $Q \varphi$ the set of states $q_{\varphi(i) j}$ and $Q \psi$ the set of states $q_{\psi(i) j}$.

1) machine does not loop in one state, neither in a set $Q \varphi$ or $Q \psi$.

- For all states of $\mathrm{A} \varphi \psi$, when the machine applies a transition staying in one state, then the head 
always goes in the same direction. But the tape is finite, on the right and on the left. Hence the machine cannot stay in the same state doing an infinite number of computation steps.

- We consider the set $\mathrm{Q} \varphi$

We stay in a state $q_{\varphi(i) j}$ if and only if $j=1$.

* if $j>1$ then we go to state $g_{p(i) j+1}$. Since $j$ has a finite number of possible values, the machine does a finite number of computation steps staying in $Q \varphi$.

* if $j=0$ then we replace a letter of $I$ by a letter of $\tilde{I}$, staying in a state of $Q \varphi$. But no transition in a state of $Q \varphi$ transforms a letter of $\bar{I}$ to a letter of $I$.Therefore the number of letter of $I$ on the tape decreases. Moreover, if there are no letter of $I$ on the tape, we cannot apply transition being in $\mathrm{q}_{\varphi(\mathrm{i}) 0}$ and staying in a state of $\mathrm{Q} \varphi$.

Hence, we do a finite number of computation steps, staying in $Q \varphi$.

- We can do the same proof for Q $\psi$.

2) The machine does not loop on $Q \varphi$ and $q_{\text {ret }}$

When it passes from $Q \varphi$ to $\mathrm{q}_{\mathrm{ret}}$, at least one letter of the tape has been overlined. Moreover, it passes from qret to $Q \varphi$ overlining one letter of the tape. Since there's a finite number of letters on the tape, there's a finite number of computation steps. We can have the same argument for Q $\psi$ and $q_{\text {ret }}^{3}$.

3) From 1) and 2) we deduce the machine loops passing by $q_{0}, q_{1}, q_{2}, q_{r e t}, q_{r e s e t}, q_{\text {again }}$, $q_{r e t}^{\prime}$, $q_{\text {reset }}, q_{\text {end }}$ and by the sets $\mathrm{Q} \varphi$ and $Q \psi$. But there is only one way to pass by $q_{0}$ : using the transition $\left(q_{\text {end }},<\right) \rightarrow\left(<, q_{0}, D\right)$. Therefore, the machine is in an instantaneous description of form $\#<\mathrm{q}_{0} \mathrm{~m}>\#$ which is, by definition, an initial configuration. Finally, according to precedent lemmas, if the machine loops from an initial configuration, then it's a proper one. $\square$ 